\section{(6) OPEN ACCESS}

\title{
Adalimumab, a human anti-TNF monoclonal antibody, outcome study for the prevention of joint damage in Japanese patients with early rheumatoid arthritis: the HOPEFUL 1 study
}

\author{
Tsutomu Takeuchi, ${ }^{1}$ Hisashi Yamanaka, ${ }^{2}$ Naoki Ishiguro, ${ }^{3}$ Nobuyuki Miyasaka, ${ }^{4}$ \\ Masaya Mukai, ${ }^{5}$ Tsukasa Matsubara, ${ }^{6}$ Shoji Uchida, ${ }^{7}$ Hideto Akama, ${ }^{8}$ \\ Hartmut Kupper, ${ }^{9}$ Vipin Arora, ${ }^{10}$ Yoshiya Tanaka ${ }^{11}$
}

\begin{abstract}
Handling editor Tore K Kvien
- Additional material is published online only. To view please visit the journal online (http://dx.doi.org/10.1136/ annrheumdis-2012-202433).

For numbered affiliations see end of article

\section{Correspondence to} Professor Tsutomu Takeuchi, Division of Rheumatology, Department of Internal Medicine, School of Medicine, Keio University,

35 Shinanomachi, Shinjuku-ku, Tokyo 160-8582, Japan; tsutake@z5.keio.jp
\end{abstract}

Received 2 August 2012 Revised 14 November 2012 Accepted 9 December 2012 Published Online First 11 January 2013

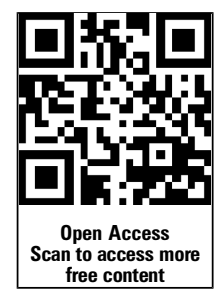

To cite: Takeuchi T, Yamanaka $\mathrm{H}$, Ishiguro $\mathrm{N}$ et al. Ann Rheum Dis 2014;73:536-543.

\section{ABSTRACT}

Objectives To evaluate the efficacy and safety of adalimumab+methotrexate (MTX) in Japanese patients with early rheumatoid arthritis (RA) who had not previously received MTX or biologics.

Methods This randomised, double-blind, placebocontrolled, multicentre study evaluated adalimumab $40 \mathrm{mg}$ every other week+MTX 6-8 mg every week versus MTX 6-8 mg every week alone for 26 weeks in patients with RA ( $\leq 2$-year duration). The primary endpoint was inhibition of radiographic progression (change $(\Delta)$ from baseline in modified total Sharp score (mTSS)) at week 26.

Results A total of 171 patients received adalimumab+MTX (mean dose, $6.2 \pm 0.8 \mathrm{mg} /$ week) and 163 patients received MTX alone (mean dose, $6.6 \pm 0.6 \mathrm{mg} /$ week, $\mathrm{p}<0.001)$. The mean RA duration was 0.3 years and $315(94.3 \%)$ had high disease activity (DAS28 $>5.1$ ). Adalimumab+MTX significantly inhibited radiographic progression at week 26 versus MTX alone ( $\Delta$ mTSS, $1.5 \pm 6.1$ vs $2.4 \pm 3.2$, respectively; $p<0.001)$. Significantly more patients in the adalimumab+MTX group (62.0\%) did not show radiographic progression $(\Delta \mathrm{mTSS} \leq 0.5)$ versus the MTX alone group $(35.4 \% ; p<0.001)$. Patients treated with adalimumab+MTX were significantly more likely to achieve American College of Rheumatology responses and achieve clinical remission, using various definitions, at 26 weeks versus MTX alone. Combination therapy was well tolerated, and no new safety signals were observed.

Conclusions Adalimumab in combination with low-dose MTX was well tolerated and efficacious in suppressing radiographic progression and improving clinical outcomes in Japanese patients with early RA and high disease activity.

\section{INTRODUCTION}

Rheumatoid arthritis (RA) is a chronic inflammatory disorder that is associated with joint damage and progressive disability, an increased risk of morbidity related to comorbid conditions, and substantial socioeconomic costs. ${ }^{1-3}$ Given the significant impact biologic therapies have had in the treatment of RA, a paradigm shift has emerged toward earlier inclusion of these therapies in the management of
RA. ${ }^{3}$ Furthermore, international guidelines published in 2010 recommend a treat-to-target goal of remission for patients with early RA in order to mitigate radiographic progression and long-term disability. ${ }^{5}$ The efficacy and safety of adalimumab, a tumour necrosis factor (TNF)- $\alpha$ inhibitor, administered as monotherapy or in combination with methotrexate (MTX) for the treatment of RA has been well established in clinical trials conducted in Western countries. ${ }^{6-12}$ In early RA, the PREMIER and OPTIMA studies demonstrated that initial combination therapy with adalimumab and MTX was superior to MTX alone in inhibiting radiographic progression and improving clinical symptoms. ${ }^{6712}$

Translating efficacy and safety results of RA Western-based studies to an Eastern populace can be potentially misleading given the genetic, medical and environmental differences (eg, body weight) observed between the two populations. ${ }^{13}$ A limited number of studies have evaluated the efficacy or effectiveness and safety of adalimumab in Japanese patients. However, these studies either assessed adalimumab monotherapy in moderate-to-severe $\mathrm{RA}^{14}$ or were retrospective $^{15}$ or postmarketing surveillance studies ${ }^{16}$ of adalimumab monotherapy or combination therapy in a population with a wide range of RA duration and prior biologic and MTX experience. Thus, a randomised, placebo-controlled study of adalimumab + MTX combination therapy in MTX-naive Japanese patients with early RA was lacking.

The current study, called adalimumab, a human anti-TNF monoclonal antibody, outcome study for the persistent efficacy under allocation to treatment strategies in early RA, or HOPEFUL 1, was conducted to compare the efficacy and safety of early intervention with adalimumab + MTX versus MTX alone for 26 weeks in inhibiting radiographic progression in MTX-naive Japanese patients with RA.

\section{PATIENTS AND METHODS}

Patients aged $\geq 20$ years were evaluated during March 2009 and November 2010 from 94 centres. Eligible patients had RA (1987-revised American College of Rheumatology (ACR) criteria), ${ }^{17}$ of $\leq 2$-year duration, a tender joint count $\geq 10$, a swollen joint count $\geq 8$, a $\mathrm{C}$ reactive protein (CRP) level $\geq 1.5 \mathrm{mg} / \mathrm{dl}$ or erythrocyte sedimentation rate 
(ESR) $\geq 28 \mathrm{~mm} / \mathrm{h}$, and had $\geq 1$ joint erosion or were rheumatoid factor positive. Patients had not previously received MTX, leflunomide or $>2$ other disease-modifying antirheumatic drugs (DMARDs). Patients who had previously received cyclophosphamide, cyclosporine, azathioprine, tacrolimus or biologic DMARDs (eg, anti-TNF- $\alpha$ therapy) and patients with a chronic infection, interstitial pneumonia, or a history of tuberculosis or malignancy were excluded from the study.

The phase III trial consisted of a randomised, double-blind, placebo-controlled, 26-week phase followed by a 26-week open-label extension phase (clinicaltrials.gov identifier, NCT00870467; only 26-week double-blind data presented). After a 4-week washout period for patients taking eligible DMARDs and a $>2$-week screening period for all patients, participants were randomised $(1: 1)$ to receive subcutaneous adalimumab $40 \mathrm{mg}$ or placebo every other week, both administered in combination with oral MTX 6-8 mg/week (adalimumab + MTX vs MTX alone) for 26 weeks. Treatment with MTX was initiated at $6 \mathrm{mg} /$ week and increased to $8 \mathrm{mg} /$ week in patients who did not experience $\geq 20 \%$ decrease from baseline in tender or swollen joint counts on or after week 8 , unless investigators indicated a safety concern. In addition, reduction of the MTX dose to $4 \mathrm{mg} /$ week was permitted at the investigator's discretion. All patients received concomitant oral folic acid $5 \mathrm{mg} /$ week. Patients who experienced a $>20 \%$ increase from baseline in tender and swollen joint counts at weeks 12,16 or 20 were to discontinue blinded treatment with adalimumab or placebo and were eligible for open-label rescue treatment with adalimumab $40 \mathrm{mg}$ every other week.

The primary endpoint was inhibition of radiographic progression assessed as the change from baseline $(\Delta)$ in modified total Sharp score (mTSS) at week 26. All single-emulsion radiographs of the hands (posteroanterior view) and feet (anteroposterior view) obtained from a patient were scored by two independent readers blinded to patient and treatment, as previously described, ${ }^{6}$ with the exception that the triquetrum/pisiform joint was not scored for erosions and the first interphalangeal joint was not scored for joint-space narrowing (range, 0-380) (see online supplementary text for more information).

Secondary efficacy endpoints included ACR responses ${ }^{18}{ }^{19}$ by visit; clinical remission (the 28-joint disease activity score with ESR (DAS28-ESR) <2.6) at week $26 ;^{20} 21$ and change from baseline in the Health Assessment Questionnaire disability index (HAQ-DI) $^{22}$ at week 26. Several additional post hoc analyses were conducted, including assessments of the DAS28-CRP, simplified disease activity index (SDAI) ${ }^{23}$ and clinical disease activity index (CDAI) scores ${ }^{24}$ over time; clinically relevant radiographic progression $(\Delta \mathrm{mTSS}>3)$; European League Against Rheumatism responses ${ }^{25}$ at week 26; and clinical remission, defined as DAS28-CRP $<2.6,{ }^{26} \mathrm{SDAI} \leq 3.3,{ }^{27}{ }^{28} \mathrm{CDAI} \leq 2.8^{28}$ or meeting Boolean remission criteria, ${ }^{27}$ at week 26 . Low, medium and high disease activity was also determined using DAS28-ESR, DAS28-CRP, SDAI and CDAI. Adverse events (AEs) and clinical laboratory parameters were routinely monitored during the study. A 28-day follow-up after the completion of or discontinuation from the study and a 70-day follow-up after the last dose of adalimumab administration were conducted to evaluate safety.

\section{Statistics}

The primary endpoint was analysed using the Wilcoxon rank sum test for observed data with a separate supportive analysis using linear extrapolation (LE) to impute missing values. Secondary endpoints were analysed using the Fisher's exact test and Wilcoxon rank sum test for discrete variables and continuous variables, respectively. Non-responder imputation was used for binary variables, and the last-observation-carried-forward approach was applied for continuous variables. The safety population included all randomised patients who received $\geq 1$ dose of study medication and had $\geq 1$ efficacy assessment.

To identify baseline predictors of no radiographic progression $(\mathrm{mTSS} \leq 0.5)$ and clinical remission (DAS28-ESR $<2.6)$,

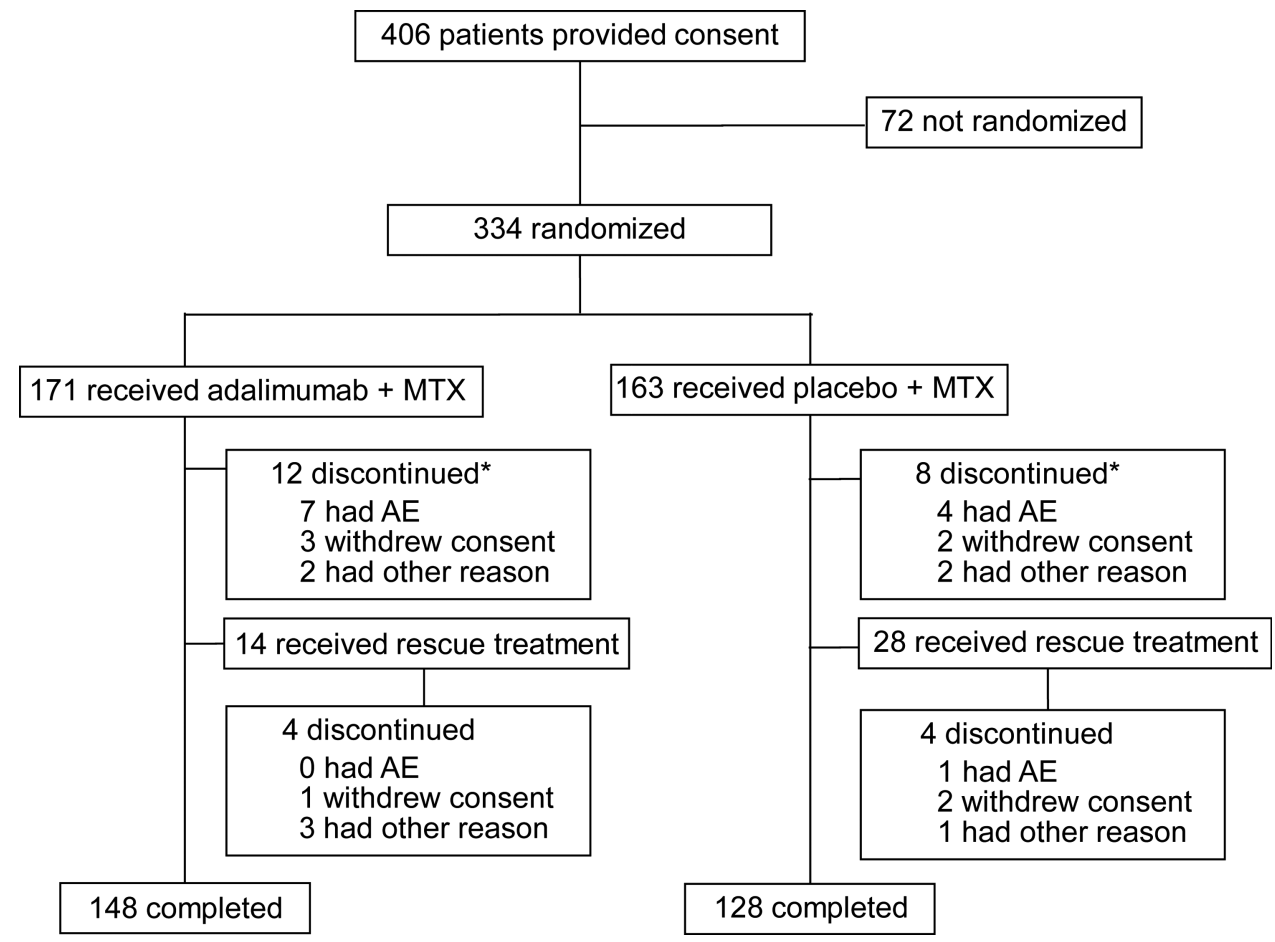

Figure 1 Patient disposition through week 26. *Three adalimumab+MTX patients and one MTX alone patient discontinued from the study by week 26; however, they were included in the efficacy analyses at week 26. AE, adverse event; MTX, methotrexate. 
univariate logistic regression analysis was performed, applying 24 baseline demographics and disease characteristics. Significant $(p<0.1)$ variables in univariate were included in multivariate models. Last, multivariate models were selected based on model fit statistics (Akaike information criterion and $r^{2}$ ) and clinical significance. Adjusted OR and 95\% CIs for selected baseline variables were calculated.

\section{RESULTS}

Overall, 334 patients were randomised to treatment and received adalimumab+MTX $(n=171)$ or MTX alone $(n=163)$, and $148(86.5 \%)$ and $128(78.5 \%)$ patients completed the double-blind portion of the study, respectively (figure 1). Demographics and baseline characteristics were well matched between treatment groups (table 1). The mean RA disease duration was 0.3 years, and the majority of patients had $\geq 1$ erosion at baseline and high disease activity. The mean MTX dose during the 26 -week study was $6.2 \pm 0.8 \mathrm{mg} /$ week in the adalimu$\mathrm{mab}+$ MTX group and $6.6 \pm 0.6 \mathrm{mg} /$ week in the MTX alone group $(p<0.001)$. After 26 weeks of treatment, $34.5 \%(59 / 171)$ of adalimumab+MTX patients were receiving MTX $8 \mathrm{mg} /$ week versus $65.0 \%(106 / 163)$ of MTX alone patients $(p<0.001)$.

\section{Radiographic progression}

Treatment with adalimumab + MTX significantly inhibited radiographic progression (figure $2 \mathrm{~A}$ ) at week 26 versus MTX alone (mean change $\pm \mathrm{SD}, \quad 1.5 \pm 6.1$ vs $2.4 \pm 3.2$, respectively; $\mathrm{p}<0.001$ ). Results were confirmed by an LE analysis (figure 2A). Changes in radiographic progression during 26 weeks of treatment were also assessed by a cumulative probability plot of $\Delta$ mTSS (figure 2B). Fewer adalimumab+MTX patients exhibited radiographic progression $(\Delta \mathrm{mTSS}>0.5)$, with $62.0 \%$ $(106 / 171)$ of patients showing no radiographic progression versus $35.4 \%(57 / 161)$ of MTX alone patients $(\mathrm{p}<0.001)$. Furthermore, only $14.0 \%$ (24/171) of adalimumab+MTX patients exhibited clinically relevant radiographic progression $(\Delta \mathrm{mTSS}>3)$ versus $37.3 \%(60 / 161)$ of MTX alone patients $(\mathrm{p}<0.001)$. In addition, a significantly higher percentage of adalimumab+MTX patients did not experience worsening $(\leq 0.5)$ in erosion score $(73.7 \%(126 / 171))$ versus MTX alone patients $(42.2 \%(68 / 161) ; \mathrm{p}<0.001)$. In patients who lacked baseline erosive damage, the continued absence of erosions was reported in more adalimumab+MTX patients versus MTX alone patients (9/9 vs 2/6 patients, respectively; $\mathrm{p}=0.01$ ).

\section{Clinical response}

A significantly higher percentage of adalimumab + MTX patients achieved ACR responses versus MTX alone patients at each assessment (figure 3A-C). Significant differences between treatment groups, observed as early as week 2, were maintained through week 26 . At week 26, a significantly larger percentage of adalimumab+MTX patients versus MTX alone patients achieved ACR20, ACR50 and ACR70 (figure 3A-C) and ACR90 (12.9\% vs 5.5\%; p=0.02) responses. Significant differences in favour of adalimumab+MTX were also observed from week 2 to 26 for DAS28-ESR, DAS28-CRP, SDAI and CDAI (see online supplementary figure $1 \mathrm{~A}-\mathrm{D}$ ). A larger percentage of adalimumab+MTX patients than MTX alone patients demonstrated good or moderate European League Against Rheumatism responses (figure 3D) and were in states of low disease activity or remission after 26 weeks of treatment (figure 3E). Furthermore, a significantly larger percentage of adalimumab+MTX patients versus MTX alone patients satisfied Boolean remission criteria (19.3\% vs $8.6 \%, p=0.007)$. Adalimumab +MTX achieved a 1.8-
Table 1 Demographics and baseline characteristics

\begin{tabular}{|c|c|c|}
\hline Parameter* & $\begin{array}{l}\text { Adalimumab+MTX } \\
(\mathrm{n}=171)\end{array}$ & MTX $(n=163)$ \\
\hline Age $\pm S D$ (year) & $54.0 \pm 13.1$ & $54.0 \pm 13.2$ \\
\hline Females (n (\%)) & $144(84.2)$ & $128(78.5)$ \\
\hline RA duration $\pm S D$ (year) & $0.3 \pm 0.4$ & $0.3 \pm 0.4$ \\
\hline Weight $\pm S D(k g)$ & $54.4 \pm 9.7$ & $56.1 \pm 12.3$ \\
\hline Previous DMARD use (n (\%)) & $74(43.3)$ & $87(53.4)$ \\
\hline 1 DMARD & $57(33.3)$ & $69(42.3)$ \\
\hline 2 DMARDs & $17(9.9)$ & $18(11.0)$ \\
\hline Corticosteroid use at baseline (n (\%)) & $58(33.9)$ & $49(30.1)$ \\
\hline RF positive (n (\%)) & $146(85.4)$ & $136(83.4)$ \\
\hline Mean titre $\pm S D(I U / m l)$ & $154.5 \pm 202.3$ & $163.7 \pm 362.8$ \\
\hline Anti-CCP positive (n (\%)) & $145(84.8)$ & $136(83.4)$ \\
\hline Mean titre $\pm \mathrm{SD}(\mathrm{U} / \mathrm{ml})$ & $386.2 \pm 694.2$ & $241.3 \pm 367.2$ \\
\hline $\operatorname{ESR}(\mathrm{mm} / \mathrm{h})$ & $59.9 \pm 30.1$ & $61.8 \pm 29.0$ \\
\hline $\mathrm{CRP}(\mathrm{mg} / \mathrm{dl})$ & $2.9 \pm 3.0$ & $3.1 \pm 3.3$ \\
\hline \multicolumn{3}{|l|}{ Swollen joint count $(n \pm S D)$} \\
\hline $0-28$ & $11.5 \pm 4.7$ & $11.8 \pm 5.3$ \\
\hline $0-66$ & $16.5 \pm 6.2$ & $17.3 \pm 7.7$ \\
\hline \multicolumn{3}{|l|}{ Tender joint count $(n \pm S D)$} \\
\hline $0-28$ & $13.2 \pm 5.8$ & $13.2 \pm 6.1$ \\
\hline $0-68$ & $20.7 \pm 9.4$ & $21.1 \pm 10.2$ \\
\hline mTSS & $13.6 \pm 22.3$ & $13.6 \pm 17.4$ \\
\hline Erosion score & $7.5 \pm 11.6$ & $7.3 \pm 9.2$ \\
\hline Joint space narrowing score & $6.2 \pm 11.4$ & $6.2 \pm 9.4$ \\
\hline DAS28-ESR & $6.6 \pm 0.9$ & $6.6 \pm 1.0$ \\
\hline DAS28-CRP & $5.8 \pm 1.0$ & $5.9 \pm 1.0$ \\
\hline HAQ-DI score & $1.1 \pm 0.7$ & $1.3 \pm 0.8$ \\
\hline SDAl score & $40.7 \pm 12.0$ & $41.4 \pm 13.8$ \\
\hline CDAI score & $37.8 \pm 10.9$ & $38.3 \pm 12.4$ \\
\hline $\begin{array}{l}\text { Physician's global assessment of } \\
\text { disease activity } \pm \text { SD }(\mathrm{mm})\end{array}$ & $65.8 \pm 18.4$ & $66.2 \pm 18.8$ \\
\hline $\begin{array}{l}\text { Patient's global assessment of disease } \\
\text { activity } \pm \text { SD (mm) }\end{array}$ & $64.1 \pm 24.8$ & $66.4 \pm 23.7$ \\
\hline \multicolumn{3}{|c|}{$\begin{array}{l}\text { *Data are mean } \pm S D \text { unless otherwise indicated. } \\
\text { CCP, cyclic citrullinated peptide; CDAl, clinical disease activity index; CRP, C reactive } \\
\text { protein; DAS28-CRP, disease activity score using a 28-joint count and CRP level; } \\
\text { DAS28-ESR, disease activity score using a 28-joint count and ESR; DMARD, } \\
\text { disease-modifying antirheumatic drug; ESR, erythrocyte sedimentation rate; HAQ-DI, } \\
\text { Health Assessment Questionnaire disability index; mTSS, modified total Sharp score; } \\
\text { MTX, methotrexate; RA, rheumatoid arthritis; RF, rheumatoid factor; SDAl, simplified } \\
\text { disease activity index. }\end{array}$} \\
\hline
\end{tabular}

to 2.2-fold increase in the percentage of patients achieving clinical remission, across all definitions of clinical remission evaluated, versus MTX alone.

A significantly larger decrease from baseline in mean HAQ-DI score, indicative of an improvement in physical function, was observed for adalimumab+MTX patients versus MTX alone patients at week $26(-0.6 \pm 0.6$ vs $-0.4 \pm 0.6 ; \mathrm{p}<0.001)$. Although the significant difference between the two groups was small (0.2 units), the percentage of patients achieving normal functionality (HAQ-DI score $<0.5$ ) after 26 weeks of treatment was also significantly higher with adalimumab+MTX (figure 3F).

\section{Factors associated with the absence of radiographic progression or with clinical remission}

Disease activity or function baseline variables generally were associated with the absence of radiographic progression $(\Delta \mathrm{mTSS} \leq 0.5)$ and with clinical remission $(\mathrm{DAS} 28-\mathrm{ESR}<2.6)$ in both treatment groups (see online supplementary text and online supplementary table 1 ). 
Figure 2 (A) Box plot of change from baseline in mTSS at week 26 with adalimumab+MTX versus MTX alone and (B) cumulative probability plot of mean change from baseline to week 26 in mTSS score (LE). Thickened horizontal lines in (A) indicate median values, the boxes mark the interval between the 25th and 75th percentiles, whiskers indicate the IQR and mean values are reported in the boxes. No radiographic progression (change from baseline in $\mathrm{mTSS} \leq 0.5$ ) was reported in $62.0 \%(106 / 171)$ of adalimumab+MTX patients versus $35.4 \%$ (57/161) of MTX alone patients $(p<0.001)$. No clinically relevant radiographic progression (change from baseline $\mathrm{mTSS} \leq 3$ ) was reported in $86.0 \%(147 / 171)$ of adalimumab+MTX patients versus $62.7 \%(101 / 161)$ of MTX alone patients $(p<0.001)(B)$. $\mathrm{LE}$, linear extrapolation; $\mathrm{mTSS}$, modified total Sharp score; MTX, methotrexate. $p$ Value determined using Wilcoxon rank sum test.
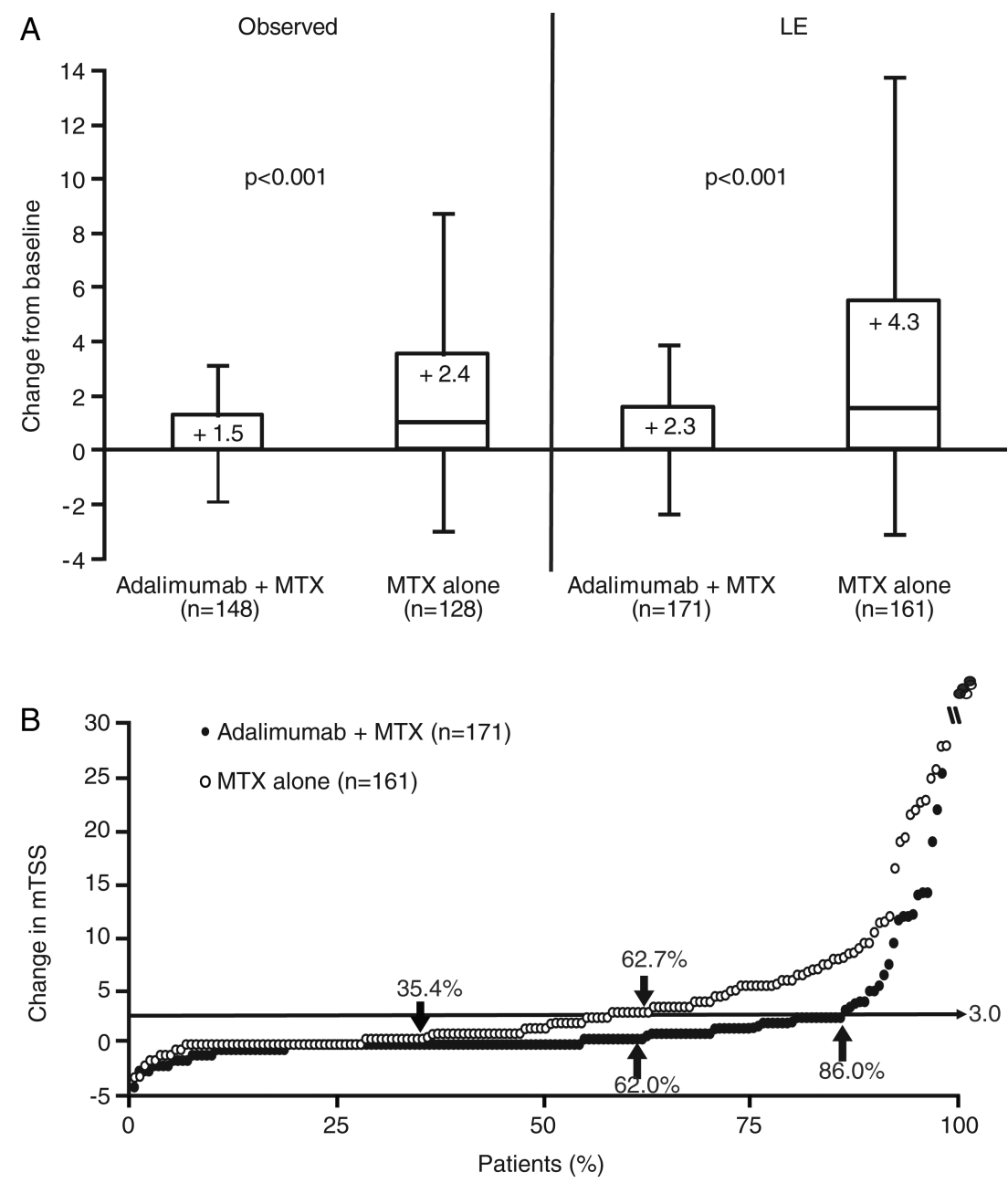

\section{Safety}

The mean treatment duration during the double-blind phase was 168.7 \pm 36.6 days for adalimumab+MTX patients (mean cumulative adalimumab dose, $477.4 \pm 104.5 \mathrm{mg}$ ) and $162.8 \pm 38.6$ days for MTX alone patients. Overall, there were 376 and 302 AEs reported in the adalimumab+MTX group and the MTX alone group, respectively. There were no significant differences in the percentage of patients with AEs in the adalimumab + MTX group (80.7\% (138/171)) versus the MTX alone group (71.8\% (117/ $163)$ ), and the incidence of severe AEs was rare (table 2). No significant differences in the incidence of AEs of interest were observed between the two groups, with the exception of injection-site reactions, which were reported in $10.5 \%$ of adalimumab+MTX patients and $3.7 \%$ of MTX alone patients $(p=0.02$; table 2$)$. Serious infections were observed in two adalimumab + MTX patients (one case each of pneumonia and infectious enteritis) and one MTX alone patient (Pneumocystis jiroveci pneumonia), occurring at rates of 2.5 and 1.4 events per 100 patient-years, respectively. There were no reports of demyelination, tuberculosis or malignancy during the study. One death, due to worsening of interstitial lung disease, occurred in the MTX alone group.

\section{DISCUSSION}

The HOPEFUL 1 study was designed to evaluate the efficacy and safety of adalimumab in combination with MTX in Japanese patients with early RA. This is the first description of a clinical trial of anti-TNF therapy+MTX versus MTX alone in MTX-naive
Japanese patients with early RA and high disease activity. It is also the first randomised trial evaluating the efficacy of anti-TNF therapy+low-dose MTX versus low-dose MTX alone for the inhibition of radiographic progression in any patient population. This study extends observations from Western studies of adalimumab by demonstrating the superiority of adalimumab+MTX to MTX alone for the inhibition of radiographic progression and improvement in clinical outcomes in Japanese patients with early RA. Moreover, the combination of adalimumab+MTX significantly improved a wide array of clinical and functional disease activity measures and responses versus MTX alone, with improvements observed as early as the first assessment (week 2) and maintained through the 26-week double-blind trial.

Following 26 weeks of treatment, the mean $\Delta$ mTSS (primary endpoint) in adalimumab+MTX patients (1.48) in the current study was significantly smaller than observed in MTX alone patients (2.38). In addition, a similar trend in inhibition of radiographic progression in patients with early RA was observed in the OPTIMA study, with a smaller mean $\triangle \mathrm{mTSS}$ in adalimumab+MTX patients $(0.15)$ versus MTX alone patients $(0.96$; $\mathrm{p}<0.001){ }^{12}$ The difference between the two treatment groups $(0.8)$ at week 26 was similar to the difference observed in the current study $\left(0.9\right.$ (observed)). ${ }^{12}$ Furthermore, baseline characteristics, including RA duration, in the two studies were generally similar, but the OPTIMA study had a lower percentage of previous DMARD use.

A similar trend in inhibition of radiographic progression in the current study was observed in the PREMIER study, with a 

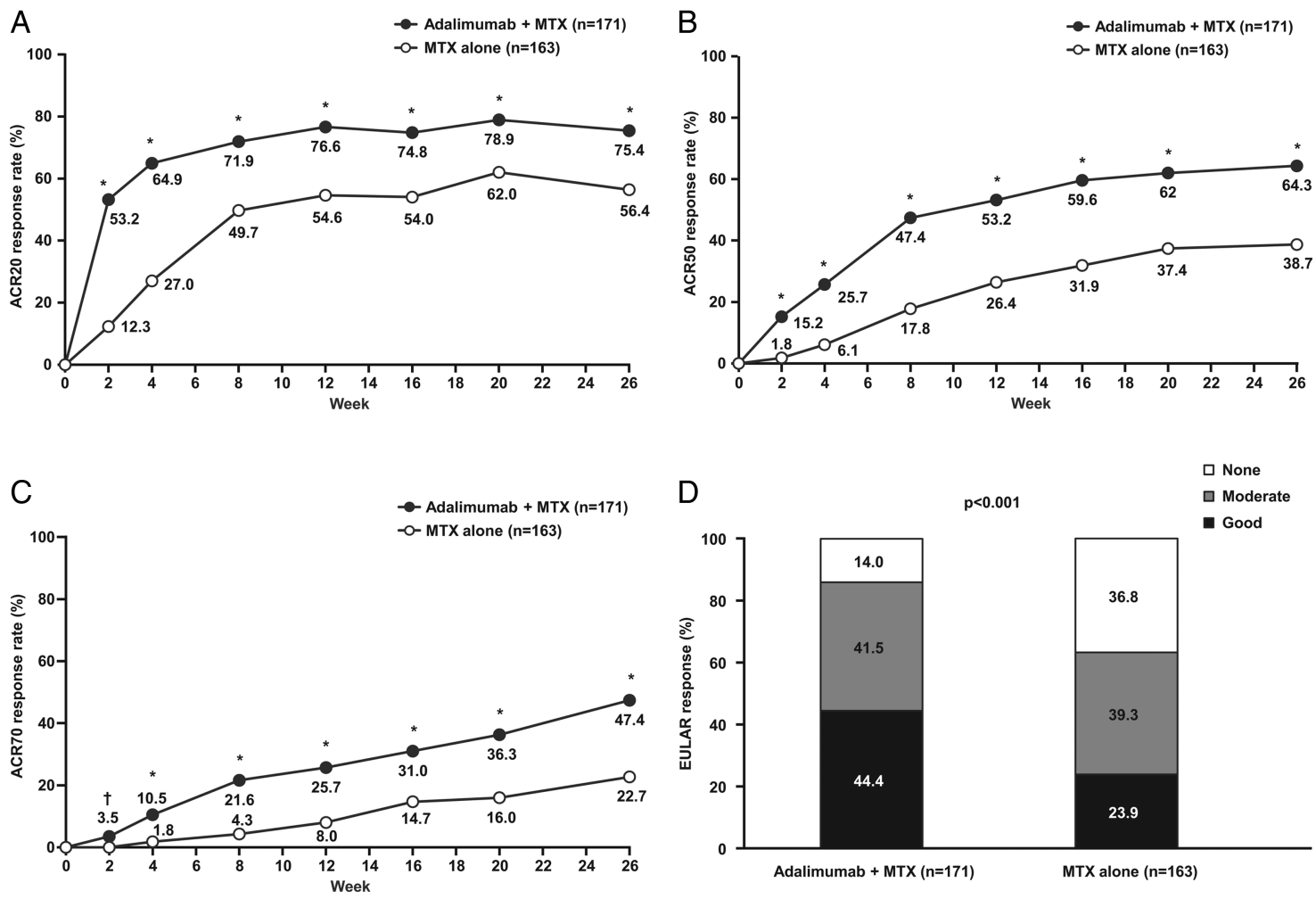

$\mathrm{E}$
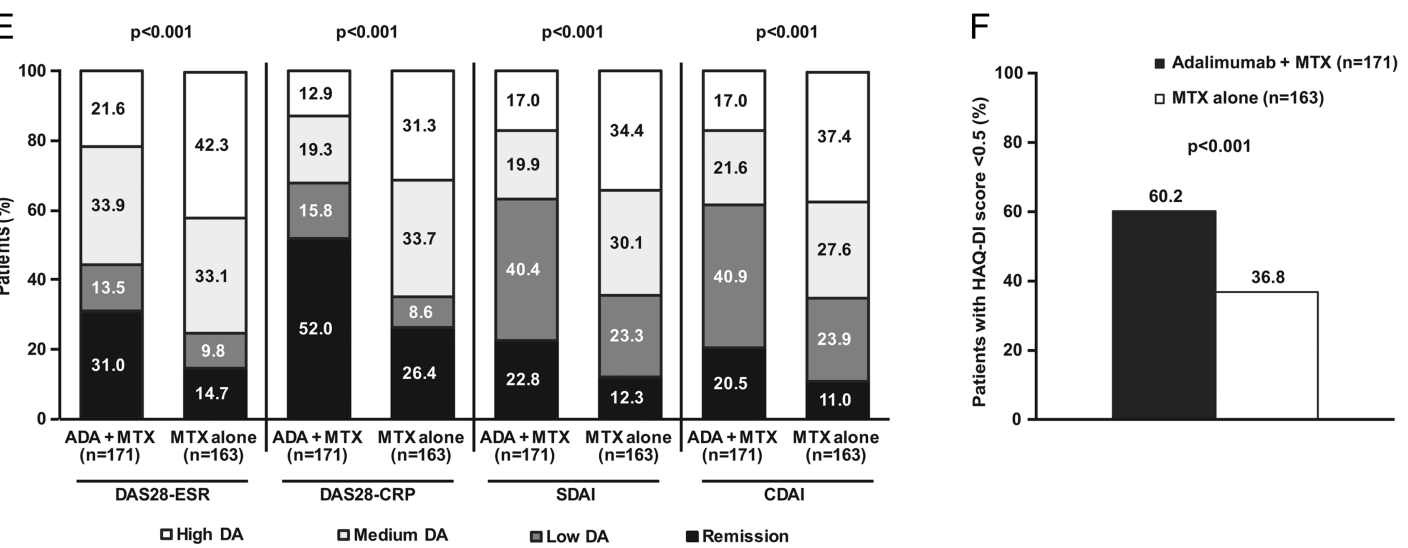

Figure 3 Percentage of patients with an (A) ACR20 response, (B) ACR50 response or (C) ACR70 response over time; (D) the percentage of patients with a EULAR response at week 26; (E) the percentage of patients with low, medium or high disease activity at week 26; and (F) the percentage of patients achieving functional remission (HAQ-DI score $<0.5$ ) at week 26 . The following values were used to identify remission, low, medium and high disease activity for each clinical assessment in (E): DAS28-ESR or DAS-CRP $(<2.6, \geq 2.6-<3.2 ; \geq 3.2-\leq 5.1,>5.1$, respectively), SDAI ( $\leq 3.3$,

$>3.3-\leq 11.0,>11.0-\leq 26.0,>26.0$, respectively), and CDAI $(\leq 2.8,>2.8-\leq 10.0,>10.0-\leq 22.0,>22.0$, respectively). * $p<0.001$ versus MTX alone. $t p=0.03$ versus MTX alone. ACR, American College of Rheumatology; ADA, adalimumab; CDAl, clinical disease activity index; DA, disease activity; DAS28-CRP, disease activity score using a 28-joint count and C reactive protein level; DAS28-ESR, disease activity score using a 28-joint count and erythrocyte sedimentation rate; EULAR, European League Against Rheumatism; HAQ-DI, Health Assessment Questionnaire disability index; MTX, methotrexate; SDAl, simplified disease activity index.

smaller mean $\Delta$ mTSS in adalimumab+MTX patients $(0.8)$ versus MTX alone patients $(3.5 ; \mathrm{p}<0.001)$. However, the mean difference in radiographic progression between the two treatments groups, although statistically significant, was smaller in the current study $(0.9$ (observed); 2.0 (LE)) than in the PREMIER study (2.7).

In the current study, the SD for the mean $\Delta$ mTSS at week 26 was generally high. When the median $\Delta$ mTSS was compared using observed data, results were in good agreement between the PREMIER study (0.0 (adalimumab+MTX) vs 1.3 (MTX alone); data on file) and the current study (0.0 (adalimumab
+MTX) vs 1.0 (MTX alone)). Alternatively, the smaller difference in improvement observed in the current study may also be related to the mTSS scoring method used, but this seems unlikely because only two joints assessed in PREMIER were omitted from scoring in the present analysis. The mean duration of RA was also shorter in the current study ( 0.3 years) versus the PREMIER study (0.7-0.8 years), although the percentage of patients who had previously taken DMARDs was higher (43.3-53.4\% vs 31.5-32.5\%). There were also slight differences in mean baseline tender and swollen joint counts and CRP levels, which were higher in the PREMIER study and considered 
Table 2 Adverse events (AEs)

\begin{tabular}{lcc}
\hline & \multicolumn{2}{c}{ Patients (n (\%)) } \\
\cline { 2 - 3 } Parameter & $\begin{array}{l}\text { Adalimumab+MTX } \\
(\mathbf{n}=171)\end{array}$ & $\begin{array}{l}\text { MTX } \\
(\mathbf{n}=163)\end{array}$ \\
\hline Any AE & $138(80.7)$ & $117(71.8)$ \\
Severe AE & $1(0.6)$ & $1(0.6)$ \\
Serious AE & $7(4.1)$ & $4(2.4)$ \\
Infectious AE & $59(34.5)$ & $48(29.4)$ \\
$\quad$ Serious infection & $2(1.2)$ & $1(0.6)$ \\
AEs leading to study drug & $7(4.1)$ & $6(3.7)$ \\
discontinuation & & \\
AEs of interest & & $21(12.9) \dagger$ \\
Elevated liver function test level & $32(18.7) \dagger$ & $6(3.7)$ \\
Injection-site reaction & $18(10.5)^{*}$ & $8(4.9)$ \\
Haematological event & $7(4.1)$ & $2(1.2)$ \\
Allergic reaction & $1(0.6)$ & $1(0.6)$ \\
Interstitial lung disease & $1(0.6)$ & $1(0.6)$ \\
Lupus-like syndrome & 0 & $1(0.6)$ \\
Opportunistic infection & 0 & \\
\hline * $p=0.02$ versus MTX. & & \\
$\dagger \geq 94 \%$ of events were mild in severity. & & \\
MTX, methotrexate. & &
\end{tabular}

related to the longer duration of RA at baseline versus the current study. Furthermore, the MTX dose of 6-8 mg/week, although consistent with the dosage commonly administered in Japan at the time the study was conducted, was substantially lower than that commonly administered in Western countries (eg, 15-20 mg/week). In the PREMIER study, MTX was initiated at $7.5 \mathrm{mg} /$ week, increased to $15 \mathrm{mg} /$ week during weeks 4-8, and increased to $20 \mathrm{mg} /$ week starting at week 9. In addition, the mean MTX dose during the 26 weeks of the current study was significantly lower in the adalimumab + MTX group $(6.2 \pm 0.8 \mathrm{mg} /$ week) versus the MTX alone group $(6.6 \pm 0.6 \mathrm{mg} /$ week; $\mathrm{p}<0.001)$, thereby potentially impacting the $\Delta \mathrm{mTSS}$ and thus the maximal difference observed between the two treatment groups. Therefore, these multiple differences may have contributed to the small difference in radiographic outcomes between the current study and the PREMIER study. Whether the difference in radiographic outcomes can be explained by differences between Japanese and Western populations remains unclear, although this seems unlikely. Longer-term studies may help elucidate potential differences in outcomes.

Since this study was conducted, the maximum approved MTX dosage in Japan has been increased from 8 to $16 \mathrm{mg} /$ week in patients with RA. Therefore, this study provides important information on the efficacy of low-dose MTX and anti-TNF therapy versus low-dose MTX alone for the inhibition of radiographic progression. Data suggest that patients with early RA who may not tolerate higher doses of MTX will likely benefit from adalimumab+low-dose MTX combination therapy.

Given the lower MTX dose prescribed, one could question whether we might only be seeing natural progression in the MTX only arm. It is ethically difficult to include a true placebo arm in clinical trials of $\geq 6$ months duration for early active RA, particularly when MTX is recommended as first-line therapy to achieve clinical remission/low disease activity. Although an important question to ponder, a placebo arm in long-term clinical trials in early active RA appears to be unrealistic, and further research using highly sensitive and reproducible imaging techniques during a short-term placebo-treatment period in early active RA is warranted.
It is also important to note that the current patient population had severe baseline symptoms, including baseline erosions, despite only several months since RA onset. This scenario is becoming increasingly less common in Western populations due to treat-to-target recommendations and earlier intervention. In Japan, general practitioners are still seeing many early RA patients and referrals to rheumatologists are often delayed. In addition, the diagnosis of RA in this trial was based upon 1987 classification criteria. Thus, these factors may have played a role in the conundrum of more severe baseline clinical symptoms yet shorter mean disease duration.

The clinical results of the current study are supported by the HARMONY study, which retrospectively determined the effectiveness and safety of adalimumab $40 \mathrm{mg}$ every other week with or without MTX (mean dose, $8.5 \mathrm{mg} / \mathrm{week}$ ) in Japanese patients with RA (mean RA duration, 9.0 \pm 9.5 years) with or without prior biologic treatment. ${ }^{15}$ Although patients in the HARMONY study had more established disease and the study design was retrospective, adalimumab+MTX patients $(n=143)$ had an improvement from baseline in DAS28-ESR score at week 24 (baseline, 5.3; week 24, 3.3), which was within the range but slightly smaller than the improvement observed in the current study at week 26 (baseline, 6.6; week 26, 3.7; see online supplementary figure 1A). Clinical remission rates for adalimumab + MTX patients were also comparable between the HARMONY study (week 24, $35.0 \%$ ) and the current study (week 26, 31.0\%).

The safety profile of the current study was generally consistent with those in previous clinical studies of adalimumab in patients with RA conducted in Japan. ${ }^{14-16}$ There were no reports of demyelination, tuberculosis or malignancy, and there were no statistically significant differences in the incidence of serious AEs, serious infections, opportunistic infections or lupus-like reactions between adalimumab+MTX patients versus MTX alone patients. There was a significantly higher incidence of injection-site reactions for adalimumab+MTX patients versus MTX alone patients, but the incidence (10.5\%) was similar to that reported for the 167 adalimumab \pm MTX patients in the HARMONY study (12.0\%). The incidence of injection-site reactions in both of these studies was lower than the $30.8 \%$ reported for the 91 adalimumab monotherapy patients $(40 \mathrm{mg}$ every other week) in the CHANGE study, ${ }^{14}$ possibly related to the immunosuppressive effects of concomitant MTX in the current study and in some of the patients in the HARMONY study.

In the multivariate regression analyses (see online supplementary table 1), lower baseline CRP level was identified as a predictor of radiographic non-progression in adalimumab+MTX patients, whereas normal baseline CRP level $(\leq 0.3 \mathrm{mg} / \mathrm{dl})$ appeared to have an increased likelihood of radiographic nonprogression. However, no baseline predictors appeared to predict both the lack of progression and clinical remission. Furthermore, baseline mTSS was not an independent predictor for either treatment group in this study.

Overall, adalimumab+MTX was well tolerated in Japanese patients with early RA with no new safety signals and with a safety and tolerability profile similar to that observed in Western populations. Administration of adalimumab in combination with MTX was efficacious in improving radiographic and clinical responses in MTX-naive patients with early RA, high disease activity and poor prognostic factors (eg, rheumatoid factor positive or with baseline erosive damage) through week 26. Given its radiographic, clinical and functional superiority versus MTX monotherapy, consideration should be given to administration 
of anti-TNF- $\alpha$ and MTX combination therapy in patients with early RA and high disease activity.

\author{
Author affiliations \\ ${ }^{1}$ Division of Rheumatology, Department of Internal Medicine, School of Medicine, \\ Keio University, Shinjuku-ku, Tokyo, Japan \\ ${ }^{2}$ Institute of Rheumatology, Tokyo Women's Medical University, Shinjuku-ku, Tokyo, \\ Japan \\ ${ }^{3}$ Department of Orthopedic Surgery, Nagoya University Graduate School and School \\ of Medicine, Showa-ku, Nagoya, Japan \\ ${ }^{4}$ Department of Medicine and Rheumatology, Graduate School of Tokyo Medical and \\ Dental University, Bunkyo-ku, Tokyo, Japan \\ ${ }^{5}$ Division of Rheumatology and Hematology, Department of Medicine, Sapporo City \\ General Hospital, Chuo-ku, Sapporo, Japan \\ ${ }^{6}$ Matsubara Mayflower Hospital, Katou-shi, Hyogo, Japan \\ ${ }^{7}$ Uchida Clinic of Rheumatic Diseases, Sumida-ku, Tokyo, Japan \\ ${ }^{8}$ Eisai Co, Ltd., Bunkyo-ku, Tokyo, Japan \\ ${ }^{9}$ Abbott GmbH \& Co KG, Ludwigshafen, Germany \\ ${ }^{10}$ Abbott Laboratories, Abbott Park, Illinois, USA \\ ${ }^{11}$ The First Department of Internal Medicine, School of Medicine, University of \\ Occupational and Environmental Health, Japan, Yahatanishi-ku, Kitakyushu, Japan
}

Acknowledgements The authors would like to thank all the patients, investigators and support staff who participated in the study, Sourav Santra, PhD, formally of Abbott, who provided statistical support, and Mary Beth C. Moncrief, PhD, of MedThink SciCom, for editorial assistance in the writing of this manuscript; this assistance was funded by Abbott.

Contributors All the authors evaluated the study results, interpreted the data and suggested additional analyses. All authors contributed to the development and critical review of manuscript and approved the final version.

Funding This study was supported by Abbott Japan Co (Tokyo, Japan) and Eisai Co (Tokyo, Japan).

Competing interests TT has received consulting fees, speaking fees, honoraria and/ or research grant support from Abbott Japan Co; Astellas Pharma Inc; Astra-Zeneca K.K. Bristol-Myers Squibb; Chugai Pharmaceutical Co; Daiichi-Sankyo Co; Eisai Co; Janssen Pharmaceutical K.K.; Mitsubishi Tanabe Pharma Corporation; Pfizer Japan Inc; and Takeda Pharmaceutical Co. HY has received research grants from Abbott Japan Co; Bristol-Myers Squibb; Chugai Pharmaceutical Co; Eisai Co; Janssen Pharmaceutical K.K.; Mitsubishi Tanabe Pharma Corporation; Otsuka Pharmaceutical Co; Pfizer Japan Inc; Takeda Industrial Pharmaceutical Co; and UCB Japan $\mathrm{Co}$, and speakers honoraria/ consulting fees from Abbott Japan Co; Bristol-Myers Squibb; Chugai Pharmaceutical Co; Eisai Co; Janssen Pharmaceutical K.K.; Mitsubishi Tanabe Pharma Corporation; Otsuka Pharmaceutical Co; Pfizer Japan Inc; Takeda Pharmaceutical Co; and UCB Japan Co. NI has received research grants from Astellas Pharmaceutical; Chugai Pharmaceutical Co; Eisai Co; and Mitsubishi Tanabe Pharmaceutical Co. NM has received research grants from Abbott Japan Co; Astellas Pharmaceutical; Banyu Pharmaceutical; Chugai Pharmaceutical Co; Daiichi Sankyo Pharmaceutical Co; Eisai Co; Janssen Pharmaceuticals; Mitsubishi Tanabe Pharma Corporation; Takeda Pharmaceutical Co; and Teijin Limited. MM has received research grants from Abbott Japan Co; Eli Lilly Japan K.K.; GlaxoSmithKline K.K.; Pfizer Japan Inc; Bristol-Myers Squibb; and Otsuka Pharmaceutical $\mathrm{Co}$, and received compensation for work on this manuscript from Abbott Japan Co. TM has received research grants from Chugai Pharmaceutical Co; Bristol-Myers Squibb; Nippon Kayaku Co; Otsuka Pharmaceutical Co; Takeda Pharmaceutical Co; Eli Lilly Japan K.K.; Eli Lilly and Company; Astellas Pharma Inc; Pfizer Japan Inc; AstraZeneca K.K.; and Santen Pharmaceutical Co, and received compensation for work on this manuscript from Abbott Japan Co. SU has received research grants from Abbott Japan Co, and received compensation for work on this manuscript from Abbott Japan Co. HA is an employee of Eisai Co, Tokyo, Japan. HK is an employee of Abbott GmbH and Co KG, Ludwigshafen, Germany, and may hold Abbott stock or options. VA is an employee of Abbott Laboratories, Abbott Park, Illinois, USA, and may hold Abbott stock or options. YT has received consulting fees, speaking fees and/or honoraria from Mitsubishi Tanabe Pharma Corporation; Abbott Japan Co; Eisai Co; Chugai Pharmaceutical Co; Janssen Pharmaceutical K.K.; Santen Pharmaceutical Co; Pfizer Japan Inc; Astellas Pharma Inc; Daiichi-Sankyo Co; GlaxoSmithKline K.K.; Astra-Zeneca; Otsuka Pharmaceutical Co; Actelion Pharmaceuticals Japan; Eli Lilly Japan K.K.; Nippon Kayaku Co; UCB Japan Co; Quintiles Transnational Japan Co; Ono Pharmaceutical Co; and Novartis Pharma K.K. YT has received research grants from Bristol-Myers Squibb; MSD K.K.; Chugai

Pharmaceutical Co; Mitsubishi Tanabe Pharma Corporation; Astellas Pharma Inc; Abbott Japan Co; Eisai Co; and Janssen Pharmaceutical K.K.

Patient consent Obtained.

Ethics approval An institutional review board approved the study at each site.

Provenance and peer review Not commissioned; externally peer reviewed.

Open Access This is an Open Access article distributed in accordance with the Creative Commons Attribution Non Commercial (CC BY-NC 3.0) license, which permits others to distribute, remix, adapt, build upon this work non-commercially, and license their derivative works on different terms, provided the original work is properly cited and the use is non-commercial. See: http://creativecommons.org/ licenses/by-nc/3.0/

\section{REFERENCES}

1 Filipovic I, Walker D, Forster F, et al. Quantifying the economic burden of productivity loss in rheumatoid arthritis. Rheumatology (Oxford) 2011; 50:1083-90.

2 Scott DL, Wolfe F, Huizinga TWJ. Rheumatoid arthritis. Lancet 2010:376:1094-108.

3 Takeuchi T. Revolutionary change in rheumatoid arthritis management with biological therapy. Keio J Med 2011;60:75-81.

4 Saag KG, Teng GG, Patkar NM, et al. American College of Rheumatology 2008 recommendations for the use of nonbiologic and biologic disease-modifying antirheumatic drugs in rheumatoid arthritis. Arthritis Rheum 2008;59:762-84.

5 Smolen JS, Aletaha D, Bijlsma JW, et al. For the T2T Expert Committee. Treating rheumatoid arthritis to target: recommendations of an international task force. Ann Rheum Dis 2010;69:631-7.

6 Breedveld FC, Weisman MH, Kavanaugh AF, et al. For the PREMIER Investigators. The PREMIER study: a multicenter, randomized, double-blind clinical trial of combination therapy with adalimumab plus methotrexate versus methotrexate alone or adalimumab alone in patients with early, aggressive rheumatoid arthritis who had not had previous methotrexate treatment. Arthritis Rheum 2006;54:26-37.

7 van der Heijde D, Breedveld FC, Kavanaugh A, et al. Disease activity, physical function, and radiographic progression after longterm therapy with adalimumab plus methotrexate: 5-year results of PREMIER. J Rheumatol 2010;37:2237-46.

8 Weinblatt ME, Keystone EC, Furst DE, et al. Adalimumab, a fully human anti-tumor necrosis factor $\alpha$ monoclonal antibody, for the treatment of rheumatoid arthritis in patients taking concomitant methotrexate: the ARMadalimumab trial. Arthritis Rheum 2003;48:35-45.

9 van de Putte LBA, Atkins C, Malaise M, et al. Efficacy and safety of adalimumab as monotherapy in patients with rheumatoid arthritis for whom previous disease modifying antirheumatic drug treatment has failed. Ann Rheum Dis 2004:63:508-16

10 Keystone EC, Kavanaugh AF, Sharp JT, et al. Radiographic, clinical, and functional outcomes of treatment with adalimumab (a human anti-tumor necrosis factor monoclonal antibody) in patients with active rheumatoid arthritis receiving concomitant methotrexate therapy: a randomized, placebo-controlled, 52-week trial. Arthritis Rheum 2004:50:1400-11.

11 Furst DE, Schiff MH, Fleischmann RM, et al. Adalimumab, a fully human anti tumor necrosis factor-alpha monoclonal antibody, and concomitant standard antirheumatic therapy for the treatment of rheumatoid arthritis: results of STAR (Safety Trial of Adalimumab in Rheumatoid Arthritis). J Rheumatol 2003;30:2563-71.

12 Kavanaugh A, Fleischmann RM, Emery $\mathrm{P}$, et al. Clinical, functional and radiographic consequences of achieving stable low disease activity and remission with adalimumab plus methotrexate or methotrexate alone in early rheumatoid arthritis: 26-week results from the randomised, controlled OPTIMA study. Ann Rheum Dis 2013:72:64-71.

13 Takeuchi T, Kameda H. The Japanese experience with biologic therapies for rheumatoid arthritis. Nat Rev Rheumatol 2010;6:644-52.

14 Miyasaka N, The CHANGE Study Investigators. Clinical investigation in highly disease-affected rheumatoid arthritis patients in Japan with adalimumab applying standard and general evaluation: the CHANGE study. Mod Rheumatol 2008;18:252-62.

15 Takeuchi T, Tanaka Y, Kaneko Y, et al. Effectiveness and safety of adalimumab in Japanese patients with rheumatoid arthritis: retrospective analyses of data collected during the first year of adalimumab treatment in routine clinical practice (HARMONY study). Mod Rheumatol 2012;22:327-38.

16 Koike $T$, Harigai M, Ishiguro $N$, et al. Safety and effectiveness of adalimumab in Japanese rheumatoid arthritis patients: postmarketing surveillance report of the first 3,000 patients. Mod Rheumatol 2012;22:498-508

17 Arnett FC, Edworthy SM, Bloch DA, et al. The American Rheumatism Association 1987 revised criteria for the classification of rheumatoid arthritis. Arthritis Rheum 1988:31:315-24.

18 Felson DT, Anderson JJ, Boers M, et al. American College of Rheumatology. Preliminary definition of improvement in rheumatoid arthritis. Arthritis Rheum 1995:38:727-35.

19 Felson DT, Anderson JJ, Lange ML, et al. Should improvement in rheumatoid arthritis clinical trials be defined as fifty percent or seventy percent improvement in core set measures, rather than twenty percent? Arthritis Rheum 1998:41:1564-70.

20 Fransen J, Creemers MCW, van Riel PLCM. Remission in rheumatoid arthritis: agreement of the disease activity score (DAS28) with the ARA preliminary remission criteria. Rheumatology (Oxford) 2004:43:1252-5

21 Prevoo ML, van't Hof MA, Kuper HH, et al. Modified disease activity scores that include twenty-eight-joint counts. Development and validation in a prospective 
longitudinal study of patients with rheumatoid arthritis. Arthritis Rheum 1995;38:44-8.

22 Fries JF, Spitz P, Kraines RG, et al. Measurement of patient outcome in arthritis. Arthritis Rheum 1980;23:137-45.

23 Smolen JS, Breedveld FC, Schiff MH, et al. A simplified disease activity index for rheumatoid arthritis for use in clinical practice. Rheumatology (Oxford) 2003:42:244-57.

24 Aletaha D, Nell VPK, Stamm T, et al. Acute phase reactants add little to composite disease activity indices for rheumatoid arthritis: validation of a clinical activity score. Arthritis Res Ther 2005;7:R796-806.

25 van Gestel AM, Prevoo MLL, van't Hof MA, et al. Development and validation of the European League Against Rheumatism response criteria for rheumatoid arthritis. Comparison with the preliminary American College of Rheumatology and the World
Health Organization/International League Against Rheumatism criteria. Arthritis Rheum 1996:39:34-40.

26 Wells G, Becker J-C, Teng J, et al. Validation of the 28-joint Disease Activity Score (DAS28) and European League Against Rheumatism response criteria based on C-reactive protein against disease progression in patients with rheumatoid arthritis, and comparison with the DAS28 based on erythrocyte sedimentation rate. Ann Rheum Dis 2009;68:954-60.

27 Felson DT, Smolen JS, Wells G, et al. American College of Rheumatology/European League Against Rheumatism provisional definition of remission in rheumatoid arthritis for clinical trials. Ann Rheum Dis 2011:70:404-13.

28 Aletaha D, Smolen J. The Simplified Disease Activity Index (SDAI) and the Clinical Disease Activity Index (CDAI): a review of their usefulness and validity in rheumatoid arthritis. Clin Exp Rheumatol 2005;23(5 suppl 39):S100-8. 